\title{
Association of CYP gene polymorphisms with breast cancer risk and prognostic factors in the Jordanian population
}

\author{
Laith N. AL-Eitan ${ }^{1,2^{*}}$ D, Doaa M. Rababa'h', Mansour A. Alghamdi ${ }^{3}$ and Rame H. Khasawneh ${ }^{4}$
}

\begin{abstract}
Background: Single nucleotide polymorphisms (SNPs) in several CYP genes have been associated with altered breast cancer (BC) risk in different populations. Despite this, there is a dearth of information on the roles of these SNPs in Jordanian BC patients. Therefore, this study aims to determine if there is any single nucleotide polymorphism (SNP) within CYP19A1, CYP2C19, CYP2C9, CYP1B1, CYP3A4, and CYP1A2 genes associated with BC in the Jordanian population. In addition, this work investigates the association between selected BC prognostic factors and variants of the aforementioned CYP candidate genes.

Methods: Blood samples were withdrawn from $221 \mathrm{BC}$ patients and 218 healthy volunteers recruited from the Jordanian population. Genomic DNA was withdrawn and, after quantification and quality control, was genotyped using the Sequenom MassARRAY ${ }^{\circledR}$ system (iPLEX GOLD). Statistical analysis was then carried out to assess allelic and genotypic frequencies as well as genetic association between cases and controls.

Results: The CYP19A1 SNP rs7176005 $(p<0.0045)$ and the CYP1A2 SNP rs762551 ( $p=0.004)$ were significantly associated with BC risk. However, no such association was found for the screened SNPs of the CYP2C9, CYP1B1, CYP2C19 and CYP3A4 genes. Regarding the prognostic factors of BC, several of the screened SNPs were associated with different pathological and clinical features.
\end{abstract}

Conclusions: Certain CYP genes, particularly CYP19A1 and CYP1A2, were associated with BC risk and development in the Jordanian population.

Keywords: Breast cancer, Prognostic factors, CYP, CYP19A1, CYP1A

\section{Background}

Several recent studies have focused on identifying breast cancer $(\mathrm{BC})$ susceptibility genes. It has been reported that pathogenic mutations account for up to $10 \%$ of worldwide BC cases [1]. The cytochrome P450 (CYPs) genes that encode for hemoproteins and have major functions in drug metabolism, are involved in the majority of metabolic and clearance processes [2]. Certain CYP genes have been implicated in cancer formation and development due to their roles in promoting oxidative stress, activating procarcinogens, and inactivating

\footnotetext{
* Correspondence: Ineitan@just.edu.jo

'Department of Applied Biological Sciences, Jordan University of Science and Technology, Irbid 22110, Jordan

${ }^{2}$ Department of Biotechnology and Genetic Engineering, Jordan University of Science and Technology, P.O. Box 3030, Irbid 22110, Jordan

Full list of author information is available at the end of the article
}

anticancer drugs [3, 4]. Not all CYP genes have an equal impact on the disease, as inherited genetic variation at the individual and population levels lead to interethnic differences in cancer risk and treatment response [5]. Examples of such genes include CYP19A1, CYP2C19, CYP2C9, CYP1B1, CYP3A4, and CYP1A2, all of which will be investigated over the course of this study.

The CYP19A1 gene encodes for the enzyme aromatase, the latter of which is targeted in $\mathrm{BC}$ therapy by aromatase inhibitors (AI) due to its critical role in estrogen biosynthesis [6]. CYP19A1 polymorphisms have been found to modulate circulating estrogen levels, alter tumor characteristics, contribute to AI-associated arthralgia, exacerbate $\mathrm{AI}$-associated bone loss, and improve letrozole efficacy in $\mathrm{BC}$ patients [7-11]. On a similar note, the CYP19A gene encodes for a hepatic enzyme that is most well-known for 
its metabolism of the antiplatelet drug clopidogrel, as polymorphisms in this gene could lead to fluctuating therapeutic effect [12]. Asian BC patients undergoing tamoxifen therapy were not impacted by CYP19A polymorphisms, but the CYP19A*17 polymorphism was associated with decreased BC risk in Caucasians [13, 14]. Furthermore, the CYP2C9 gene is responsible for a substantial proportion of phase I metabolism, but its highly polymorphic nature gives rise to changes in metabolic activity and potential adverse drug reactions [15]. In tamoxifentreated $\mathrm{BC}$ patients, CYP2C9 polymorphisms were thought to influence rates of disease-free survival as well as tumor characteristics, but no significant association was found in the Asian population $[13,16]$.

Although its role in drug metabolism is not as clearly delineated, the overexpression of CYP1B1 in tumour cells gives credence to its importance in cancer research [17]. In American and Chinese women, CYP1B1 polymorphisms were not associated with $\mathrm{BC}$ risk, but, in combination with other factors, certain CYP1B1 alleles were related to $\mathrm{BC}$ risk in the Finnish-Caucasian and Turkish populations [18-21]. In contrast, the CYP3A4 gene is heavily involved in the deactivation and biotransformation of one-third of clinically used drugs [22]. In the context of $\mathrm{BC}$, the CYP3A4*1B polymorphism was significantly associated with early onset of puberty, the latter of which increases $\mathrm{BC}$ risk [23]. Moreover, the CYP3A4 gene was found to inactivate the antineoplastic drug docetaxel and potentially compound its side effects $[24,25]$. Lastly, the CYP1A2 gene is known to metabolize a number of procarcinogens as well as anticancer drugs (like tamoxifen), and its activity is influenced by dietary patterns [26]. The CYP1A2 AA genotype was found to strip the protective effect of caffeine against $\mathrm{BC}$ from patients, while patients with the CYP1A2"1F AA genotype experienced slower ER-positive tumor growth upon coffee consumption [27, 28].

The primary aim of the present study was to determine whether any association existed between certain single nucleotide polymorphisms (SNPs) of the CYP19A1, CYP2C19, CYP2C9, CYP1B1, CYP3A4, and $C Y P 1 A 2$ genes and $\mathrm{BC}$ in the Jordanian population. A secondary objective investigated the association between selected BC prognostic factors and variants of the aforementioned CYP candidate genes.

\section{Methods}

\section{Study cohort}

Female participants were recruited from the Jordanian population at the Jordanian Royal Medical Hospital after obtaining written informed consent. Ethical approval for the present study was obtained from the Institutional Review Board (IRB) at Jordan University of Science and Technology.
A total of 473 blood samples were collected from unrelated Jordanian women including 231 healthy volunteers and 242 were diagnosed with breast cancer. Both the cases and controls were randomly chosen and adjusted to be matched with regard to age, sex, and ethnic origin.

\section{Extraction of genomic DNA}

$5 \mathrm{ml}$ of blood were withdrawn from each volunteer and subject to DNA extraction procedures using the DNA Purification KitWizard ${ }^{\bullet}$ Genomic (Promega, USA). The purified DNA underwent quality control testing via agarose gel electrophoresis (to check for integrity) and measured by the Nano-Drop ND-1000 UV-Vis Spectrophotometer (BioDrop, UK).

\section{Genotyping}

Multiplex PCR was used to amplify Loci of candidate SNPs followed by a primer extention process (Mass EXTEND) resulting in allele-specific DNA products. Mass spectrometry was used for minisequencing reaction product analysis. Afterward, the extension PCR products were separated onto a 384 well spectroCHIP and placed into the MALDI-TOF (Matrix Assisted Laser Desorption/Ionization Time-of-Flight) mass spectrometer. Finally, a software system (Spectro TYPER-RT (RT for real-time) was used to analyze the results. SNPs were genotyped using Sequenom iPLEX (Sequenom, San Diego, CA, USA). MassARRAY assay design (version 3.1) software (Sequenom MassARRAY system) was used to design the PCR in addition to the single base extension primers (SBE) at the AGRF; Australian Genome Research Facility (AGRF) (Melbourne, Australia).

\section{Statistical analysis}

The equation for the Hardy-Weinberg equilibrium (HWE), $p^{2}+2 p q+q^{2}=1$, was used for all SNPs in both groups using the Chi-square $\left(x^{2}\right)$ goodness of-fit test.Variations between cases and controls were calculated by employing Pearson's chi-squared using the Statistical Package for the Social Sciences (SPSS), version 25.0 (SPSS, Inc., Chicago, IL). The odds ratio (OR) was also calculated using binary logistic regression with 95\% confidence intervals (CI).On the other hand, genetic association analysis using different genetic models was carried out in this study, $p$-values were considered to be statistically significant only if they were less than 0.05 . Haploview program (version 4.2) was used to perform haplotype analysis test for linkage disequilibrium (LD) and to allow for the analysis of LD blocks and haplotype.

\section{Correction for multiple testing}

Multiple comparison method of $\mathrm{Li}$ and Ji (2005) was used to estimate the effective number of SNPs $\left(N_{e m}\right)$ [29], which 
employs a modification of an earlier approach by Nyholt (2004) [30]. Modified Bonferroni procedure was applied to determine a target alpha level $\left(0.05 / N_{e m}\right)$ that would maintain an overall significance level of 0.0045 or less.

\section{Results}

\section{Samples characteristics}

General characteristics of the study cohort in the current research was summarized and categorized by AL-Eitan et al., (2017) [31]. All controls were unrelated healthy females from Jordan with approximate average age of $50.8 \pm 12.6$ years and their ages ranged from 24 to 90 years.

Data obtained for this study was available for 221 female patients who were diagnosed with BC. All participants were unrelated with averages of ages at BC diagnosis (51.1 \pm 16.5$)$, at pregnancy $(22.6 \pm 2)$, at menarche $(13.8 \pm 0)$ and at menopause $(48.31 \pm 4.5)$. The estimated average of body mass index (BMI) for patients group was $31.28 \pm 3.48$. In addition, $66 \%$ of patients have practiced breastfeeding while $34 \%$ of them did not breastfed at any stage of their life.

Other clinical features were also investigated, co-morbidities, $46 \%$ of BC patients suffered from other complications such as hypertension, coronary artery disease, asthma, and diabetes mellitus. Furthermore $32 \%$ of patients have family history with BC disease while $27 \%$ of the patients had allergy. However, life style such as smoking was included in this analysis, we found that only $30 \%$ of the cases were smokers.

In terms of $\mathrm{BC}$ pathological features, $80 \%$ of all cases had been diagnosed with invasive ductal carcinoma compared to the $20 \%$ of patients who found with in-situ ductal carcinoma. With regard to hormone receptor status, estrogen and progesterone receptors were found on the malignant cells of 74 and $44 \%$ of patients, respectively, while $40 \%$ of patients were positive for (human epidermal growth factor receptor 2) (HER2) expression. Certain pathological features such as nodal involvement, have been investigated, $82 \%$ of cases were reported with lymph node involvement while $48.4 \%$ of the cases showed axillary lymph node metastatic. In addition, patients were grouped into two categories depending on the differentiation rate; low and mild differentiation (62\%) and highly differentiated tumor (38\%). likewise, stages of tumor progression for patients were divided into two group grade 1, 2 (PT1 + PT2; 90.3\%) and grade 3, 4. (PT3 + PT4; 9.7\%). Molecular subtyping of BC depending on estrogen, progesterone, and HER2 status to divide the disease into subtypes: luminal $\mathrm{A}(\mathrm{ER}(+)$ and /or $\mathrm{PR}(+)$ Her2 (-)), luminal $\mathrm{B}(\mathrm{ER}(+)$ and /or $\mathrm{PR}(+)$ Her2 $(+))$, triple negative(ER(-) and /or PR(-) Her2 (-)). Finally, In this study, $47 \%$ of patients were L.A, $41 \%$ were L.B while $12 \%$ were T.N [32].

\section{Quality control (QC)}

Table 1 illustrates the investigated SNPs and their information, in addition the table shows the genotype call rates that ranged from 96 to $97 \%$. After quality control, 222 patient out of 242 and 218 controls out of 231 were included in the analysis.

\section{Minor allelic frequency of the investigated CYP450 candidate gene SNPs}

Six CYP450 genes essential to drug metabolism were included in this study. Table 2 displays the investigated SNPs within these genes and the allelic distribution frequency for each gene's minor allele as well as the HWE $p$-value. In this study one SNP (rs408611) of CYP2C9 did not fulfil HWE and was excluded $(P=0.036<0.05)$ from the genetic analysis.

\section{Association of CYP450 SNPs with breast cancer (BC)}

The influence of the selected polymorphisms within the CYP19A1, CYP2C9, CYP2C19, CYP1B1, CYP3A4, and $C Y P 1 A 2$ genes on $B C$ in Jordanian population was investigated via a genetic association analysis between cases and controls. Table 3 shows the frequency distribution for both the alleles and the genotypes in the cases and controls. For most of the SNPs, the frequencies of the variant alleles were slightly higher in the cases than in the controls. For example, $23 \%$ of the cases carried the variant allele $\mathrm{T}$ of the CYP19A1 SNP rs7176005 compared to $15 \%$ of the controls.

The CYP19A1 SNPs rs7176005 and rs6493497 were significantly associated with $\mathrm{BC}$ in terms of both their alleles and genotypes ( $p$-value $<0.05)$. According to our findings the variant allele $(\mathrm{T})$ of rs7176005 was significantly higher among cases (23\%) than it among controls (15\%). In similar way, rs6493497 variant allele (A) found more frequent among patients than it within controls. We suggest that the variants alleles of CYP19A1 SNPs; rs7176005 and rs6493497 could be an influence factors for increasing the breast cancer risk.

In addition, the rs762551 SNP of the CYP1A2 gene was also found to be significantly associated with $\mathrm{BC}$ with regard to its genotype ( $p$-value $=0.00426207)$ but not to its allele ( $p$-value $=0.2250304$ ). Our results revealed that the variant allele $(C)$ of rs762551 among controls was higher than it among cases. Furthermore, the CC genotype among cases (23\%) was less than it among controls (38). However according to our statistical significant result, we propose that the CC genotype of the rs762551 SNP of the CYP1A2 gene may act as protective factor against breast cancer progression and development.

No significant correlation was found between the rest of the screened SNPs of the CYP2C9, CYP1B1, CYP2C19 and $C Y P 3 A 4$ genes and breast cancer ( $p$-value>0.05). 
Table 1 List of genes, their SNPs, positions, and genotyping data based on the whole cohort $(N=449)$

\begin{tabular}{|c|c|c|c|c|c|}
\hline Gene & SNP_ID & Position $^{a}$ & SNP & SNP Location & Assay pass rate \\
\hline \multirow[t]{5}{*}{$\overline{C Y P 19 A 1}$} & rs7176005 & 15:51339082 & $C>T^{M A}$ & 2 KB Upstream Variant & $97 \%$ \\
\hline & rs6493497 & 15:51338638 & $G>A^{M A}$ & 2 KB Upstream Variant & $96 \%$ \\
\hline & rs700519 & 15:51215771 & $G>A^{M A}$ & Missense Variant & $97 \%$ \\
\hline & rs10046 & 15:51210789 & $G>A^{M A}$ & 3 Prime UTR Variant & $96 \%$ \\
\hline & rs4646 & 15:51210647 & $A^{M A}>C$ & 3 Prime UTR Variant & $97 \%$ \\
\hline \multirow[t]{2}{*}{ CYP2C9 } & rs1799853 & 10:94942290 & $\mathrm{C}>\mathrm{T}^{\mathrm{MA}}$ & Missense Variant & $96 \%$ \\
\hline & rs4086116 & 10:94947445 & $C>T^{M A}$ & Intron Variant & $96 \%$ \\
\hline CYP2C19 & rs4244285 & 10:94781859 & $G>A^{M A}$ & Synonymous Variant & $94 \%$ \\
\hline CYP1B1 & rs10175368 & 2:38080719 & $C>T^{M A}$ & Ningle Nucleotide Variant & $96 \%$ \\
\hline CYP3A4 & rs35599367 & 7:99768693 & $G>A^{M A}$ & Intron Variant & $97 \%$ \\
\hline CYP1A2 & rs762551 & $15: 74749576$ & $C^{M A}>A$ & Intron Variant & $97 \%$ \\
\hline
\end{tabular}

MA minor allele

${ }^{a}$ Chromosome positions are based on NCBI Human Genome Assembly Build

${ }^{b}$ Ratio of the number of discordant genotypes to the number of duplicates

Different genetic models have been incorporated into the genetic association analysis in this study. Table 4 summarizes these different models (which included dominant, additive, and recessive genetic models) and shows the chi-squared values differentiating between the cases and controls. The CYP19A1 SNP rs7176005 was significantly associated with breast cancer for both the Rare $\mathrm{Hz}(\mathrm{TT})$ vs Het $(\mathrm{CT})\left(x^{2}=\right.$ 4.57) and the Rare $\mathrm{Hz}(\mathrm{TT})$ vs Common $\mathrm{Hz}(\mathrm{CC})\left(\chi^{2}=8.44\right)$ genetic models. Similarly, the CYP19A1 SNP rs6493497 was also associated with $\mathrm{BC}$ for both of the aforementioned genetic models. In addition, a connection between the CYP1A2 SNP rs762551 and $\mathrm{BC}$ was observed for the Rare $\mathrm{Hz}(C C)$ vs Het $(\mathrm{AC})$ genetic model $\left(\chi^{2}=4.92\right)$. No such link to $B C$ was found for any of the other investigated SNPs using these genetic models.

\section{Association of CYP450 SNPs with breast cancer (BC) prognostic factors}

The prognostic factors of $\mathrm{BC}$ can be broadly categorized into clinical and pathological features. Clinical features encompass factors like body mass index, smoking, co-morbidity, and age at first $\mathrm{BC}$ diagnosis, while pathological features involve progesterone and estrogen receptor statuses, tumor stage, and lymph node involvement, among others. Table 5 demonstrates the relationship between a number of different clinical and pathological features of $\mathrm{BC}$ and the investigated CYP19A1 SNPs. The CYP19A1 SNPs rs10046 and rs4646 were found to be significantly associated with age at first $\mathrm{BC}$ diagnosis $(p$-value $=0.007)$ and lymph node involvement $(p$-value $=0.022)$. In contrast, the

Table 2 Minor allele frequencies among breast cancer patients and healthy controls and the HWEC P-value of CYP450 candidate gene polymorphisms

\begin{tabular}{|c|c|c|c|c|c|c|c|}
\hline \multirow[t]{2}{*}{ Gene } & \multirow[t]{2}{*}{ SNP ID } & \multicolumn{3}{|c|}{ Cases $(n=221)$} & \multicolumn{3}{|c|}{ Controls $(n=218)$} \\
\hline & & MA & $\mathrm{MAF}^{\mathrm{a}}$ & HWE $^{\mathrm{b}} p$-value & MA & MAF & HWE $p$-value \\
\hline \multirow[t]{5}{*}{ CYP19A1 } & rs7176005 & $\mathrm{T}$ & 0.23 & 0.85 & $\mathrm{~T}$ & 0.15 & 0.18 \\
\hline & rs6493497 & A & 0.22 & 0.56 & A & 0.15 & 0.18 \\
\hline & rs700519 & A & 0.03 & 0.68 & A & 0.02 & 0.75 \\
\hline & rs10046 & A & 0.42 & 0.27 & A & 0.44 & 0.50 \\
\hline & rs4646 & A & 0.29 & 0.33 & A & 0.29 & 0.25 \\
\hline \multirow[t]{2}{*}{ CYP2C9 } & rs1799853 & T & 0.14 & 0.084 & T & 0.15 & 0.063 \\
\hline & rs4086116 & T & 0.22 & 0.17 & T & 0.23 & 0.036 \\
\hline CYP2C19 & rs4244285 & $A$ & 0.11 & 0.74 & A & 0.08 & 0.84 \\
\hline CYP1B1 & rs10175368 & T & 0.29 & 0.87 & T & 0.26 & 0.16 \\
\hline CYP3A4 & rs35599367 & A & 0.02 & 0.70 & $A$ & 0.03 & 0.70 \\
\hline CYPIAZ & rs762551 & C & 0.33 & 0.88 & C & 0.37 & 0.10 \\
\hline
\end{tabular}

MA minor allele

N/A: not applicable

aMA: minor allele frequency

${ }^{b}$ HWE: Hardy-Weinberg equilibrium 
Table 3 Association of the investigated CYP450 candidate gene polymorphisms and breast cancer (BC)

\begin{tabular}{|c|c|c|c|c|c|c|c|c|}
\hline \multirow[t]{2}{*}{ Gene } & \multirow[t]{2}{*}{ SNP ID } & \multicolumn{5}{|c|}{ Allelic and Genotypic Frequencies in Cases and Controls } & \multirow[b]{2}{*}{ Odd Ratio } & \multirow[b]{2}{*}{$\mathrm{Cl}(95 \%)$} \\
\hline & & Allele/Genotype & $\begin{array}{l}\text { Cases: total number }= \\
222 \\
\mathrm{n}(\%)\end{array}$ & $\begin{array}{l}\text { Controls: total number = } \\
218 \\
\mathrm{n}(\%)\end{array}$ & $P$-value* & $\begin{array}{l}\text { Pearson Chi- } \\
\text { square }\end{array}$ & & \\
\hline \multirow[t]{24}{*}{ CYP19A1 } & \multirow[t]{5}{*}{ rs7176005 } & C & $341(0.77)$ & $370(0.85)$ & \multirow[t]{2}{*}{0.002} & \multirow[t]{2}{*}{9.408} & \multirow[t]{2}{*}{0.584} & \multirow[t]{2}{*}{$0.4132-0.8254$} \\
\hline & & $\mathrm{T}$ & $101(0.23)$ & $64(0.15)$ & & & & \\
\hline & & $\mathrm{CC}$ & 132(0.6) & 155(0.71) & \multirow[t]{3}{*}{0.003} & \multirow[t]{3}{*}{11.06} & \multirow[t]{3}{*}{5.3538} & \multirow[t]{3}{*}{$1.1497-24.9301$} \\
\hline & & $C T$ & $77(0.35)$ & $60(0.28)$ & & & & \\
\hline & & $\pi$ & $12(0.05)$ & $2(0.01)$ & & & & \\
\hline & \multirow[t]{5}{*}{ rs6493497 } & G & $343(0.78)$ & $368(0.85)$ & \multirow[t]{2}{*}{0.005} & \multirow[t]{2}{*}{7.57} & \multirow[t]{2}{*}{0.615} & \multirow[t]{2}{*}{$0.4341-0.8711$} \\
\hline & & A & $97(0.22)$ & $64(0.15)$ & & & & \\
\hline & & GG & $12(0.05)$ & $2(0.01)$ & \multirow[t]{3}{*}{0.008} & \multirow[t]{3}{*}{9.627} & \multirow[t]{3}{*}{5.7049} & $1.2236-26.5984$ \\
\hline & & GA & 73(0.33) & $60(0.28)$ & & & & \\
\hline & & AA & $135(0.61)$ & $154(0.71)$ & & & & \\
\hline & rs700519 & G & $430(0.97)$ & $425(0.98)$ & 0.534 & 0.385 & 0.7588 & $0.3164-1.8196$ \\
\hline & & A & $12(0.03)$ & $9(0.02)$ & & & & \\
\hline & & $A G$ & $12(0.05)$ & $9(0.04)$ & 0.530 & 0.394 & 0.7536 & $0.3109-1.8266$ \\
\hline & & GG & $209(0.95)$ & 208(0.96) & & & & \\
\hline & rs10046 & G & $252(0.58)$ & $243(0.56)$ & 0.537 & 0.381 & 1.0883 & $0.8318-1.424$ \\
\hline & & A & $182(0.42)$ & $191(0.44)$ & & & & \\
\hline & & AA & $34(0.16)$ & $42(0.19)$ & 0.585 & 1.071 & 0.6577 & $0.3764-1.1493$ \\
\hline & & GA & $114(0.53)$ & $107(0.49)$ & & & & \\
\hline & & GG & $69(0.32)$ & $68(0.31)$ & & & & \\
\hline & rs4646 & $C$ & $313(0.71)$ & $309(0.71)$ & $\mathrm{N} / \mathrm{A}$ & N/A & 0.9972 & $0.7454-1.3342$ \\
\hline & & A & $129(0.29)$ & $127(0.29)$ & & & & \\
\hline & & AA & $22(0.1)$ & $22(0.1)$ & 0.996 & 0.008 & 0.9683 & $0.4867-1.9264$ \\
\hline & & CA & $85(0.38)$ & 83(0.38) & & & & \\
\hline & & CC & $114(0.52)$ & $113(0.52)$ & & & & \\
\hline CYP2C9 & rs1799853 & C & $377(0.86)$ & $367(0.85)$ & 0.424 & 0.638 & 1.1665 & $0.7991-1.7029$ \\
\hline & & T & $59(0.14)$ & $67(0.15)$ & & & & \\
\hline & & CC & $166(0.76)$ & 159(0.73) & 0.752 & 0.569 & 0.8187 & $0.2772-2.4179$ \\
\hline & & $C T$ & $45(0.21)$ & $49(0.23)$ & & & & \\
\hline & & $\pi$ & $7(0.03)$ & $9(0.04)$ & & & & \\
\hline CYP2C19 & rs4244285 & G & $383(0.89)$ & $390(0.92)$ & 0.060 & 3.515 & 0.6413 & $0.4019-1.0233$ \\
\hline & & A & $49(0.11)$ & $32(0.08)$ & & & & \\
\hline & & $A A$ & $3(0.01)$ & $1(0.0)$ & 0.170 & 3.543 & 2.175 & $0.2152-21.9809$ \\
\hline & & $A G$ & $43(0.2)$ & $30(0.14)$ & & & & \\
\hline & & GG & $170(0.79)$ & $180(0.85)$ & & & & \\
\hline CYP1B1 & rs10175368 & $C$ & $310(0.71)$ & $318(0.74)$ & 0.350 & 0.87 & 0.8682 & $0.6452-1.1684$ \\
\hline & & $\mathrm{T}$ & $128(0.29)$ & $114(0.26)$ & & & & \\
\hline & & CC & $109(0.5)$ & $121(0.56)$ & 0.340 & 2.156 & 0.7297 & $0.3512-1.5163$ \\
\hline & & $C T$ & $92(0.42)$ & $76(0.35)$ & & & & \\
\hline & & $\pi$ & 18(0.08) & 19(0.09) & & & & \\
\hline CYPЗА4 & rs35599367 & G & $431(0.98)$ & $425(0.97)$ & 0.974 & 0.001 & 1.0141 & $0.435-2.3642$ \\
\hline & & A & $11(0.02)$ & $11(0.03)$ & & & & \\
\hline & & GA & $11(0.05)$ & $11(0.05)$ & 0.974 & 0.001 & 1.0145 & $0.4304-2.3914$ \\
\hline
\end{tabular}


Table 3 Association of the investigated CYP450 candidate gene polymorphisms and breast cancer (BC) (Continued)

\begin{tabular}{|c|c|c|c|c|c|c|c|c|}
\hline \multirow[t]{2}{*}{ Gene } & \multirow[t]{2}{*}{ SNP ID } & \multicolumn{5}{|c|}{ Allelic and Genotypic Frequencies in Cases and Controls } & \multirow[b]{2}{*}{ Odd Ratio } & \multirow[b]{2}{*}{ Cl (95\%) } \\
\hline & & Allele/Genotype & $\begin{array}{l}\text { Cases: total number }= \\
222 \\
\mathrm{n}(\%)\end{array}$ & $\begin{array}{l}\text { Controls: total number = } \\
218 \\
\mathrm{n}(\%)\end{array}$ & $P$-value* & $\begin{array}{l}\text { Pearson Chi- } \\
\text { square }\end{array}$ & & \\
\hline & & GG & $210(0.95)$ & $207(0.95)$ & & & & \\
\hline \multirow[t]{5}{*}{ CYPIA2 } & rs762551 & A & 295(0.67) & $274(0.63)$ & 0.225 & 1.472 & 1.188 & $0.8993-1.5695$ \\
\hline & & C & $145(0.33)$ & $160(0.37)$ & & & & \\
\hline & & AA & $98(0.45)$ & $95(0.44)$ & 0.004 & 10.916 & 0.3712 & $0.1968-0.7003$ \\
\hline & & $A C$ & $99(0.45)$ & $84(0.39)$ & & & & \\
\hline & & CC & 23(0.1) & 38(0.18) & & & & \\
\hline
\end{tabular}

Cl confidence interval

${ }^{*}$-Value $<0.0045$ considered as significant

Table 4 Genetic association analysis for the investigated CYP450 candidate gene polymorphisms using different genetic models

\begin{tabular}{|c|c|c|c|c|c|}
\hline Gene & SNP ID & Category Test & Odds Ratio & $95 \% \mathrm{Cl}$ & Chi square \\
\hline \multirow[t]{15}{*}{ CYP19A1 } & \multirow[t]{3}{*}{ rs7176005 } & Het (CT) vs Common Hz (CC) & 1.51 & $1-2.27$ & 3.87 \\
\hline & & Rare Hz (TT) vs Het (CT) & 4.68 & $1.01-21.69$ & 4.57 \\
\hline & & Rare Hz (TT) vs Common Hz (CC) & 7.05 & $1.55-32.05$ & 8.44 \\
\hline & \multirow[t]{3}{*}{ rs6493497 } & Het (AG) vs Common Hz (GG) & 1.39 & $0.92-2.1$ & 2.43 \\
\hline & & Rare $\mathrm{Hz}(\mathrm{AA})$ vs Het (AG) & 4.93 & $1.06-22.9$ & 4.94 \\
\hline & & Rare $\mathrm{Hz}(\mathrm{AA})$ vs Common Hz (GG) & 6.84 & $1.5-31.13$ & 8.13 \\
\hline & \multirow[t]{3}{*}{ rs700519 } & Het (AG) vs Common Hz (GG) & 1.33 & $0.55-3.22$ & 0.39 \\
\hline & & Rare $\mathrm{Hz}(\mathrm{AA})$ vs Het (AG) & NA & NA & NA \\
\hline & & Rare $\mathrm{Hz}(\mathrm{AA})$ vs Common $\mathrm{Hz}(\mathrm{GG})$ & NA & NA & NA \\
\hline & \multirow[t]{3}{*}{ rs10046 } & Het (AG) vs Common Hz (GG) & 1.05 & $0.69-1.61$ & 0.05 \\
\hline & & Rare $\mathrm{Hz}(\mathrm{AA})$ vs Het (AG) & 0.76 & $0.45-1.28$ & 1.06 \\
\hline & & Rare $\mathrm{Hz}(\mathrm{AA})$ vs Common Hz (GG) & 0.8 & $0.45-1.4$ & 0.62 \\
\hline & \multirow[t]{3}{*}{ rs4646 } & Het (AC) vs Common Hz (CC) & 1.02 & $0.68-1.51$ & 0.01 \\
\hline & & Rare Hz (AA) vs Het (AC) & 0.98 & $0.5-1.9$ & 0 \\
\hline & & Rare $\mathrm{Hz}(\mathrm{AA})$ vs Common $\mathrm{Hz}(\mathrm{CC})$ & 0.99 & $0.52-1.89$ & 0 \\
\hline \multirow[t]{3}{*}{ CYP2C9 } & \multirow[t]{3}{*}{ rs1799853 } & Het (CT) vs Common Hz (CC) & 0.88 & $0.56-1.39$ & 0.3 \\
\hline & & Rare $\mathrm{Hz}(\mathrm{TT})$ vs Het $(\mathrm{CT})$ & 0.85 & $0.29-2.46$ & 0.09 \\
\hline & & Rare $\mathrm{Hz}(\mathrm{TT})$ vs Common Hz (CC) & 0.74 & $0.27-2.05$ & 0.33 \\
\hline \multirow[t]{3}{*}{ CYP2C19 } & \multirow[t]{3}{*}{ rs4244285 } & Het (AG) vs Common Hz (GG) & 1.52 & $0.91-2.53$ & 2.58 \\
\hline & & Rare $\mathrm{Hz}(\mathrm{AA})$ vs Het (AG) & 2.09 & $0.21-21.1$ & 0.41 \\
\hline & & Rare $\mathrm{Hz}(\mathrm{AA})$ vs Common $\mathrm{Hz}(\mathrm{GG})$ & 3.18 & $0.33-30.84$ & 1.11 \\
\hline \multirow[t]{3}{*}{ CYP1B1 } & \multirow[t]{3}{*}{ rs10175368 } & Het (CT) vs Common Hz (CC) & 1.34 & $0.9-2$ & 2.11 \\
\hline & & Rare $\mathrm{Hz}(\mathrm{TT})$ vs Het $(\mathrm{CT})$ & 0.78 & $0.38-1.6$ & 0.46 \\
\hline & & Rare Hz (TT) vs Common Hz (CC) & 1.05 & $0.53-2.11$ & 0.02 \\
\hline \multirow[t]{3}{*}{ CYP1A2 } & \multirow[t]{3}{*}{ rs762551 } & Het (AC)vs Common Hz (AA) & 1.14 & $0.76-1.71$ & 0.42 \\
\hline & & Rare Hz (CC) vs Het(AC) & 0.51 & $0.28-0.93$ & 4.92 \\
\hline & & Rare $\mathrm{Hz}(\mathrm{CC})$ vs Common $\mathrm{Hz}(\mathrm{AA})$ & 0.59 & $0.33-1.06$ & 3.18 \\
\hline
\end{tabular}


Table 5 Association between different CYP19A1 SNP genotypes and the clinico-pathological features of breast cancer (BC)

\begin{tabular}{|c|c|c|c|c|c|}
\hline \multirow[t]{2}{*}{ Clinical features } & \multicolumn{5}{|l|}{ CYP19A1 } \\
\hline & rs10046 & rs7176005 & rs700519 & rs6493497 & rs4646 \\
\hline $\begin{array}{l}\text { Body mass index }{ }^{b} \\
\mathrm{n}=221\end{array}$ & 0.510 & 0.570 & 0.811 & 0.547 & 0.092 \\
\hline $\begin{array}{l}\text { Age at first pregnancy }{ }^{b} \\
n=195\end{array}$ & 0.122 & 0.200 & 0.825 & 0.200 & 0.956 \\
\hline $\begin{array}{l}\text { Age at } \mathrm{BC} \text { diagnosis }{ }^{\mathrm{b}} \\
n=221\end{array}$ & 0.007 & 0.681 & 0.836 & 0.789 & 0.921 \\
\hline $\begin{array}{l}\text { Allergy }^{a} \\
\mathrm{n}=221\end{array}$ & 0.936 & 0.935 & 0.401 & 0.967 & 0.856 \\
\hline $\begin{array}{l}\text { Age at menarche }{ }^{b} \\
n=221\end{array}$ & 0.989 & 0.421 & 0.044 & 0.405 & 0.383 \\
\hline $\begin{array}{l}\text { Breastfeeding status }{ }^{a} \\
n=221\end{array}$ & 0.401 & 0.095 & 0.367 & 0.127 & 0.721 \\
\hline $\begin{array}{l}\text { Age at menopause }{ }^{b} \\
n=108\end{array}$ & 0.290 & 0.736 & 0.002 & 0.736 & 0.797 \\
\hline $\begin{array}{l}\text { Family history } \\
\mathrm{n}=221\end{array}$ & 0.571 & 0.121 & 0.927 & 0.138 & 0.123 \\
\hline $\begin{array}{l}\text { Co-morbidity } \\
\mathrm{n}=221\end{array}$ & 0.104 & 0.557 & 0.924 & 0.566 & 0.905 \\
\hline $\begin{array}{l}\text { Smoking a } \\
n=216\end{array}$ & 0.166 & 0.607 & 0.059 & 0.580 & 0.617 \\
\hline \multicolumn{6}{|l|}{ Pathological features } \\
\hline $\begin{array}{l}\text { Progesterone receptor status }{ }^{a} \\
n=198\end{array}$ & 0.157 & 0.754 & 0.867 & 0.719 & 0.755 \\
\hline $\begin{array}{l}\text { Estrogen receptor status }{ }^{a} \\
n=191\end{array}$ & 0.714 & 0.463 & 0.159 & 0.339 & 0.227 \\
\hline $\begin{array}{l}\text { Human epidermal growth factor receptor } 2 \text { marker (HER2) } \\
n=139\end{array}$ & 0.945 & 0.445 & 0.493 & 0.485 & 0.468 \\
\hline $\begin{array}{l}\text { Heteromolecular BC markers }{ }^{\mathrm{a}} \\
n=138\end{array}$ & 0.510 & 0.788 & 0.915 & 0.797 & 0.754 \\
\hline $\begin{array}{l}\text { Tumor differentiation }{ }^{\text {a }} \\
n=197\end{array}$ & 0.273 & 0.851 & 0.918 & 0.860 & 0.906 \\
\hline $\begin{array}{l}\text { Axillary lymph nodes }{ }^{\text {a }} \\
\mathrm{n}=221\end{array}$ & 0.844 & 0.793 & 0.584 & 0.687 & 0.297 \\
\hline $\begin{array}{l}\text { Tumor stage }{ }^{a} \\
n=208\end{array}$ & 0.491 & 0.118 & 0.922 & 0.080 & 0.236 \\
\hline $\begin{array}{l}\text { Histology classification }{ }^{\text {a }} \\
n=209\end{array}$ & 0.722 & 0.475 & 0.407 & 0.507 & 0.320 \\
\hline $\begin{array}{l}\text { Tumor size } \\
n=208\end{array}$ & 0.726 & 0.547 & 0.879 & 0.472 & 0.448 \\
\hline $\begin{array}{l}\text { Lymph node involvement }{ }^{a} \\
\mathrm{n}=221\end{array}$ & 0.953 & 0.681 & 0.617 & 0.691 & 0.022 \\
\hline
\end{tabular}

a Pearson's chi-squared test was used to determine genotype-phenotype association

${ }^{\mathrm{b}}$ Analysis of variance (ANOVA) test was used to determine genotype-phenotype association

$P$-Value $<0.0045$ considered as significant

CYP19A1 SNP rs700519 was significantly associated with both the age at menopause $(p$-value $=0.002)$ and age at menarche $(p$-value $=0.04)$.

Table 6 shows the relationship between the same BC prognostic factors and the CYP2C9, CYP1A2, CYP3A4 and $C Y P 2 C 19$ SNPs. None of the clinical or pathological features of $\mathrm{BC}$ were associated with the $C Y P 2 C 9$ SNPs. However, the rs762551 of the CYP1A2 gene was found to be significantly associated with age at menopause $(p$-value $=0.034)$, Human epidermal growth factor receptor 2 marker (HER2) ( $p$-value $=0.028)$, histology classification $(p$-value $=0.011)$, and lymph involvement $(p$-value $=0.001)$ but not with any of the clinical features. Likewise, the CYP3A4 SNP rs35599367 was significantly associated with age at first pregnancy $(p$-value $=0.009)$ and tumor stage $(p$-value $=$ 
Table 6 Association between different CYP2C9, CYP1A2, CYP3A4 and CYP2C19 SNP genotypes and the clinico-pathological features of breast cancer $(\mathrm{BC})$

\begin{tabular}{|c|c|c|c|c|c|}
\hline Clinical features & CYP2C9 & CYP1A2 & CYP1B1 & CYP3A4 & CYP2C19 \\
\hline & rs1799853 & rs762551 & rs 10175368 & rs35599367 & rs4244285 \\
\hline $\begin{array}{l}\text { Body mass index }{ }^{b} \\
n=221\end{array}$ & 0.078 & 0.178 & 0.552 & 0.286 & 0.834 \\
\hline $\begin{array}{l}\text { Age at first pregnancy } \\
\mathrm{n}=195\end{array}$ & 0.144 & 0.292 & 0.179 & 0.032 & 0.785 \\
\hline $\begin{array}{l}\text { Age at } B C \text { diagnosis }{ }^{b} \\
n=221\end{array}$ & 0.511 & 0.675 & 0.295 & 0.391 & 0.132 \\
\hline $\begin{array}{l}\text { Allergy } \\
\mathrm{n}=221\end{array}$ & 0.721 & 0.927 & 0.859 & 0.146 & 0.920 \\
\hline $\begin{array}{l}\text { Age at menarche }{ }^{b} \\
n=221\end{array}$ & 0.834 & 0.738 & 0.905 & 0.869 & 0.931 \\
\hline $\begin{array}{l}\text { Breastfeeding status }{ }^{a} \\
n=221\end{array}$ & 0.250 & 0.450 & 0.196 & 0.009 & 0.161 \\
\hline $\begin{array}{l}\text { Age at menopause }{ }^{b} \\
n=108\end{array}$ & 0.356 & 0.034 & 0.554 & 0.161 & 0.266 \\
\hline $\begin{array}{l}\text { Family history } \\
n=221\end{array}$ & 0.562 & 0.167 & 0.521 & 0.494 & 0.294 \\
\hline $\begin{array}{l}\text { Co-morbidity } \\
\mathrm{n}=221\end{array}$ & 0.659 & 0.456 & 0.774 & 0.822 & 0.349 \\
\hline $\begin{array}{l}\text { Smoking } \\
n=216\end{array}$ & 0.305 & 0.705 & 0.899 & 0.341 & 0.528 \\
\hline \multicolumn{6}{|l|}{ Pathological features } \\
\hline $\begin{array}{l}\text { Progesterone receptor status }{ }^{a} \\
\mathrm{n}=198\end{array}$ & 0.213 & 0.118 & 0.378 & 0.554 & 0.213 \\
\hline $\begin{array}{l}\text { Estrogen receptor status }{ }^{\text {a }} \\
\mathrm{n}=191\end{array}$ & 0.409 & 0.208 & 0.286 & 0.511 & 0.409 \\
\hline $\begin{array}{l}\text { Human epidermal growth factor receptor } 2 \text { marker (HER2) } \\
\mathrm{n}=139\end{array}$ & 0.495 & 0.028 & 0.109 & 0.566 & 0.495 \\
\hline $\begin{array}{l}\text { Heteromolecular BC markers } \\
\mathrm{n}=138\end{array}$ & 0.691 & 0.043 & 0.024 & 0.914 & 0.081 \\
\hline $\begin{array}{l}\text { Tumor differentiation }{ }^{\text {a }} \\
\mathrm{n}=197\end{array}$ & 0.285 & 0.577 & 0.498 & 0.734 & 0.285 \\
\hline $\begin{array}{l}\text { Axillary lymph nodes }{ }^{\text {a }} \\
\mathrm{n}=221\end{array}$ & 0.956 & 0.587 & 0.179 & 0.346 & 0.956 \\
\hline $\begin{array}{l}\text { Tumor stage }{ }^{a} \\
n=208\end{array}$ & 0.743 & 0.153 & 0.469 & 0.048 & 0.743 \\
\hline $\begin{array}{l}\text { Histology classification }{ }^{\text {a }} \\
n=209\end{array}$ & 0.708 & 0.011 & 0.238 & 0.118 & 0.708 \\
\hline $\begin{array}{l}\text { Tumor size }{ }^{b} \\
n=208\end{array}$ & 0.407 & 0.433 & 0.991 & 0.318 & 0.407 \\
\hline $\begin{array}{l}\text { Lymph node involvement }{ }^{a} \\
n=221\end{array}$ & 0.194 & 0.001 & 0.406 & 0.516 & 0.194 \\
\hline
\end{tabular}

a Pearson's chi-squared test was used to determine genotype-phenotype association

${ }^{\mathrm{b}}$ Analysis of variance (ANOVA) test was used to determine genotype-phenotype association

$P$-Value $<0.0045$ considered as significant

0.04). Lastly, the CYP2C19 SNP rs4244285 was significantly correlated with HER2 ( $p$-value $=0.02)$.

The heteromolecular BC markers PR, ER, and HER2 were also investigated to their recently elucidated prognostic and predictive roles in the disease. $\mathrm{BC}$ can be divided into three classifications based on marker expression: Luminal A $(\mathrm{ER}(+)$ and /or $\mathrm{PR}(+)$ and HER2-neu (-)), Luminal $\mathrm{B}(\mathrm{ER}(+)$ and /or $\mathrm{PR}(+)$ and HER2-neu(+)), and Triple Negative (ER(-), PR(-) and HER2-neu $(-)$ ). (Tables 5 and 6) show the relationship between each of these $\mathrm{BC}$ classifications and the investigated SNPs. The CYP1B1 SNP rs10175368 
and the CYP1A2 SNP rs762551 both showed an association with the different $\mathrm{BC}$ classifications, with $p$ values of 0.024 and 0.043 , respectively.

\section{Haplotypic analysis}

Haplotype was further studied as a part of the genetic association analysis, our findings revealed a strong linkage disequilibrium between five CYP19A1 SNPs (rs10046, rs4646, rs6493497, rs700519 and rs7176005) that formed one block. However, no significant association was found between any of these haplotypes and $\mathrm{BC}$ in Jordanian Arabs (Table 7).

\section{Discussion}

Polymorphisms in the CYP genes have been reported to modulate the risk and development of breast cancer (BC) as well as individual response to anticancer drugs $[3,4]$. As previously mentioned, the present study investigated the role of certain CYP19A1, CYP2C9, CYP2C19, CYP1B1, CYP3A4, and CYP1A2 polymorphisms on BC in Jordanian patients and healthy volunteers.

With regard to CYP19A1 polymorphisms, rs7176005 and rs6493497 result in variable response to aromataseinhibitor treatment in early-stage BC patients, while another study found that these polymorphisms did not increase estrogen biosynthesis in postmenopausal women $[33,34]$. In a meta-analysis of over 20,000 cases and controls, it was found that rs10046 on its own did not increase $\mathrm{BC}$ risk [35]. A systematic review of a dozen studies reported that the CYP19A1 SNP rs4646 conferred a beneficial effect in that it increased the progression time in patients with metastatic BC [36]. Similarly, the rs700519 SNP of the CYP19A1 gene was not significantly associated with $\mathrm{BC}$ in any ethnic or mixed population in one meta-analysis, but the rs700519 SNP was significantly associated with $\mathrm{BC}$ susceptibility in the Han Chinese population [37, 38]. In contrast, our findings show that only the CYP19A1 SNPs rs7176005 and rs6493497 were significantly associated with $\mathrm{BC}$ in the Jordanian population. However, sample selection bias may be encountered in this study. Even though the patients were randomly selected to avoid potential bias,
$46 \%$ of $\mathrm{BC}$ patients suffered from other complications such as hypertension, coronary artery disease, asthma, and diabetes mellitus that might influence the results. On the other hand, we found that the CYP19A1 SNPs rs7176005 and rs10046 were significantly associated with body mass index and age at $\mathrm{BC}$ diagnosis, while rs700519 was linked to both age at menopause and age at menarche.

With regard to CYP2C9, the rs1799853 SNP was found to be significantly associated with cyclophosphamide toxicity when analyzed as a part of a haplotype [39]. However, the rs1799853 SNP was not associated with BC in Asian Singaporeans, nor was it associated with increased $\mathrm{BC}$ risk in women undergoing menopausal hormone therapy [13, 40]. In our study, none of the investigated CYP2C9 SNPs were significantly associated with $\mathrm{BC}$ or its prognostic factors in Jordanian females. On the other hand, the CYP2C19 SNP rs4244285 was not associated with BC risk nor with estrogen levels in the German population, but it was associated with longer $\mathrm{BC}$ survival rates compared to the wild type $[14,41]$. In the Thai population, the rs4244285 SNP was the most common variant but it was not associated with tamoxifen efficiency, while, in the Dutch population, this SNP was significantly linked to time tamoxifen failure [42, 43]. Similar to the CYP2C9 SNPs, our findings illustrate that the CYP2C19 SNP rs4244285 is not associated with BC risk or any of its prognostic factors in Jordanians.

In terms of CYP1A2, the rs762551 polymorphism was not associated with $\mathrm{BC}$ risk or modulated estrogen levels in the German populations, but it resulted in altered BC risk in the Thai population $[40,44,45]$. According to two different meta-analyses, the CYP1A2 SNP rs762551 was associated with cancer susceptibility in the Caucasian but not the Asian or mixed populations [46, 47]. However, another meta-analysis comprising 17,600 cases and controls found no significant association between rs762551 and BC development in any ethnic population [48]. Our findings show that, in Jordanian BC patients, the CYP1A2 SNP rs762551 was not associated with $B C$ risk, but it was significantly related to the following $\mathrm{BC}$ prognostic factors: age at menopause, histological class,

Table 7 Association between breast cancer (BC) and different haplotypes

\begin{tabular}{|c|c|c|c|c|}
\hline $\begin{array}{l}\text { Haplotype CYP19A1 Block (rs10046, rs4646, } \\
\text { rs6493497,rs700519 and rs7176005) }\end{array}$ & $\begin{array}{l}\text { Frequency of } \\
\text { block }\end{array}$ & $\begin{array}{l}\text { Frequency ratio \% (case: } \\
\text { control) }\end{array}$ & $\begin{array}{l}\text { Odds ratio }(95 \%) \\
\mathrm{Cl}\end{array}$ & $\begin{array}{l}P \text { - } \\
\text { value }\end{array}$ \\
\hline ACGGC & 0.348 & 0.3189:0.3765 & 1 & N/A \\
\hline GAGGC & 0.2543 & 0.428 I: 0.2662 & $1.12(0.78-1.62)$ & 0.54 \\
\hline GCGGC & 0.1914 & 0.191: 0.1927 & $1.16(0.77-1.75)$ & 0.48 \\
\hline ACAGT & 0.0818 & 0.099: 0.0645 & $1.88(0.94-3.76)$ & 0.076 \\
\hline GCAGT & 0.0612 & $0.0677: 0.0544$ & $1.69(0.83-3.47)$ & 0.15 \\
\hline
\end{tabular}

Global haplotype association $p$ - value:0.066

N/A not applicable

$P$-Value $<0.05$ considered as significant 
HER2 marker status, and lymph node involvement. This polymorphism was also significantly associated with the heteromolecular marker classes L.A vs L.B. vs T.N.

Concerning the CYP3A4, the rs35599367 SNP was significantly associated with exemestane concentration in postmenopausal BC patients, nor was it associated with dose reduction or peripheral neuropathy in paclitaxeltreated BC patients $[49,50]$. However, it was associated with higher everolimus concentrations in the blood of postmenopausal BC patients [51]. The findings of the present study show that the CYP3A4 SNP rs35599367 was not associated with $\mathrm{BC}$ risk, but it was significantly associated with age at first pregnancy and tumor stage. Regarding the $C Y P 1 B 1$, it has been previously reported that rs10175368 has negligible influence on BC risk in the Caucasian and Polish population [52, 53]. Correspondingly, our findings showed no significant association between the CYP1B1 SNP rs10175368 and BC risk or $\mathrm{BC}$ prognostic factors, but an association was found between this SNP and the different BC heteromolecular classifications.

This study can enrich the scarce literature about breast cancer and its implication in the Arab world and in Jordan particularly. In addition, identifying prognostic factors that can predict the risk of cancer development and progression is in demand in clinical practice. In this study, we hypothesized that the genetic markers that potentially induce breast cancer development and progression are different from those involved in breast cancer subtypes and prognosis. Therefore, determining variants that involved in breast cancer prognosis can help in stratifying patients in clinical trials and lead to characterizing the most effective therapy to provide patients with personalized medicine.

On the other hand, a case-control study with a small sample number could lead to selection bias which was the main limitation in this study. However, other potential limitations were avoided; no bias is caused by population stratification, as the Jordanian Arab population is a relatively homogenous population. Moreover, there were no significant difference between case and control groups in term of basic demographic characteristics. In addition, gender bias was excluded as all the participants limited to females. Finally, genotyping in this study was done using the Sequenom MassARRAY ${ }^{\bullet}$ system, which is one of the most error-free, high throughput, accurate, sensitive, and robust sequencing techniques.

\section{Conclusions}

Conclusively, the findings of the current study suggest that certain polymorphisms of the CYP19A1 and CYP1A2 genes are implicated in BC risk and development in Jordanian patients. Furthermore, several CYP genes have been found to be significantly associated with
$\mathrm{BC}$ prognostic factors, resulting in potentially worsened prognoses for carriers of those polymorphisms. However, this study is an exploratory one that, having identified potential BC susceptibility gene polymorphisms, leaves room for future studies to corroborate these findings using a larger sample size.

\begin{abstract}
Abbreviations
AGRF: Australian Genome Research Facility; BC: Breast Cancer (BC); CYP: Cytochrome P450 (CYP); DNA: Deoxyribonucleic acid (DNA); ER: Estrogen Receptor; HER2: Human epidermal growth factor receptor 2 marker; Het: Heterozygote; HWE: Hardy-Weinberg equilibrium (HWE); Hz: Homozygote; IRB: Institutional Review Board; JRMS: Jordanian Royal Medical Services; PR: Progesterone Receptor; SNPs: Single nucleotide polymorphisms; SPSS: Statistical Package for the Social Sciences; $x^{2}$ : Chi squared value $\left(X^{2}\right)$
\end{abstract}

\section{Acknowledgements}

The authors thank the Jordanian Royal Medical Services (JRMS), Amman, Jordan, for approving this study in the first instance and making the clinical data and samples available for the study.

\section{Authors' contributions}

LNA-E designed the method study and supervised the study. LNA-E, DMR and MAA lead the implementation of the method, performed the data analysis and drafted the manuscript. LNA-E, DMR, MAA and RHK helped with the interpretation, and description of the results. All authors read and approved the final manuscript.

\section{Funding}

This study was funded by the Deanship of Research (RN: 20140204), Jordan University of Science and Technology. There is no role for JUST in the design of the study; collection, analysis, and interpretation of data; and in writing the manuscript.

\section{Availability of data and materials}

The data sets generated and/or analyzed over the course of the study are not publicly available but are available from the corresponding author on reasonable request.

\section{Ethics approval and consent to participate}

All procedures performed in studies involving human participants were in accordance with the ethical standards of the Institutional Review Board (IRB) at Jordan University of Science and Technology with ethical code number (14/78/2014). Written informed consent was obtained from all individual participants included in the study.

\section{Consent for publication}

Not applicable.

\section{Competing interests}

The authors declare that they have no competing interests.

\section{Author details}

'Department of Applied Biological Sciences, Jordan University of Science and Technology, Irbid 22110, Jordan. ${ }^{2}$ Department of Biotechnology and Genetic Engineering, Jordan University of Science and Technology, P.O. Box 3030, Irbid 22110, Jordan. ${ }^{3}$ College of Medicine, King Khalid University, Abha, Saudi Arabia. ${ }^{4}$ Department of Hematopathology, King Hussein Medical Center (KHMC), Jordan Royal Medical Services (RMS), Amman 11118, Jordan.

Received: 25 February 2019 Accepted: 26 August 2019

Published online: 02 September 2019

\section{References}

1. Apostolou P, Fostira F. Hereditary breast cancer: the era of new susceptibility genes. Biomed Res Int. 2013;747318. https://doi.org/1 $0.1155 / 2013 / 747318$.

2. Guengerich FP. Cytochrome P450 and chemical toxicology. Chem Res Toxicol. 2008;21:70-83. https://doi.org/10.1021/tx700079z. 
3. Rodriguez-Antona C, Ingelman-Sundberg M. Cytochrome P450 pharmacogenetics and cancer. Oncogene. 2006;25:1679-91. https://doi. org/10.1038/sj.onc.1209377.

4. Hrycay EG, Bandiera SM. Involvement of Cytochrome P450 in Reactive Oxygen Species Formation and Cancer. In: Advances in pharmacology (San Diego, Calif.); 2015. p. 35-84.

5. Zhou Y, Ingelman-Sundberg M, Lauschke VM. Worldwide distribution of cytochrome P450 alleles: a meta-analysis of population-scale sequencing projects. Clin Pharmacol Ther. 2017;102:688-700. https://doi.org/10.1002/ cpt.690.

6. Chumsri S, Howes T, Bao T, et al. Aromatase, aromatase inhibitors, and breast cancer. J Steroid Biochem Mol Biol. 2011;125:13-22. https://doi.org/1 0.1016/J.JSBMB.2011.02.001.

7. Haiman CA, Dossus L, Setiawan WW, et al. Genetic variation at the CYP19A locus predicts circulating estrogen levels but not breast cancer risk in postmenopausal women. Cancer Res. 2007;67:1893-7. https://doi.org/10.115 8/0008-5472.CAN-06-4123.

8. Fasching PA, Loehberg CR, Strissel PL, et al. Single nucleotide polymorphisms of the aromatase gene (CYP19A1), HER2/neu status, and prognosis in breast cancer patients. Breast Cancer Res Treat. 2008;112: 89-98. https://doi.org/10.1007/s10549-007-9822-2.

9. Mao JJ, Su HI, Feng R, et al. Association of functional polymorphisms in CYP19A1 with aromatase inhibitor associated arthralgia in breast cancer survivors. Breast Cancer Res. 2011;13:R8. https://doi.org/10.11 86/bcr2813.

10. Napoli N, Rastelli A, Ma C, et al. Genetic polymorphism at Val80 (rs700518) of the CYP19A1 gene is associated with aromatase inhibitor associated bone loss in women with ER (+) breast cancer. Bone. 2013;55:309-14. https://doi.org/10.1016/J.BONE.2013.04.021.

11. Colomer R, Monzo M, Tusquets I, et al. A single-nucleotide polymorphism in the aromatase gene is associated with the efficacy of the aromatase inhibitor letrozole in advanced breast carcinoma. Clin Cancer Res. 2008;14: 811-6. https://doi.org/10.1158/1078-0432.CCR-07-1923.

12. Dean L. Clopidogrel therapy and CYP2C19 genotype. In: Pratt V, McLeod H, Dean L, et al., editors. Medical genetic Summaries. Bethesda: National Center for Biotechnology Information; 2012.

13. Lim JSL, Chen XA, Singh O, et al. Impact of CYP2D6, CYP3A5, CYP2C9 and CYP2C19 polymorphisms on tamoxifen pharmacokinetics in Asian breast cancer patients. Br J Clin Pharmacol. 2011;71:737-50. https://doi.org/1 0.1111/j.1365-2125.2011.03905.x.

14. Justenhoven C, Hamann U, Pierl CB, et al. CYP2C19*17 is associated with decreased breast cancer risk. Breast Cancer Res Treat. 2009;115:391-6. https://doi.org/10.1007/s10549-008-0076-4

15. Van Booven D, Marsh S, McLeod H, et al. Cytochrome P450 2C9-CYP2C9. Pharmacogenet Genomics. 2010;20:277-81. https://doi.org/10.1097/FPC. 0b013e3283349e84.

16. Jernström H, Bågeman E, Rose C, et al. CYP2C8 and CYP2C9 polymorphisms in relation to tumour characteristics and early breast cancer related events among 652 breast cancer patients. Br J Cancer. 2009;101:1817-23. https:// doi.org/10.1038/sj.bjc.6605428.

17. Murray Gl, Melvin WT, Greenlee WF, Burke MD. Regulation, function, and tissue-specific expression of cytochrome P450 CYP1B1. Annu Rev Pharmacol Toxicol. 2001;41:297-316. https://doi.org/10.1146/annurev.pharmtox.41.1.297.

18. De Vivo I, Hankinson SE, Li L, et al. Association of CYP1B1 polymorphisms and breast cancer risk. Cancer Epidemiol Biomark Prev. 2002;11:489-92

19. Wen W, Cai Q, Shu X-O, et al. Cytochrome P450 1B1 and catechol-Omethyltransferase genetic polymorphisms and breast Cancer risk in Chinese women: results from the Shanghai breast Cancer study and a meta-analysis. Cancer Epidemiol Biomark Prev. 2005;14:329-35. https://doi.org/10.1158/1 055-9965.EPI-04-0392.

20. Sillanpää P, Heikinheimo L, Kataja V, et al. CYP1A1 and CYP1B1 genetic polymorphisms, smoking and breast cancer risk in a Finnish Caucasian population. Breast Cancer Res Treat. 2007;104:287-97. https://doi.org/10.1 007/s10549-006-9414-6.

21. Kocabas N, SardaS S, Cholerton S, et al. Cytochrome P450 CYP1B1 and catechol $\mathrm{O}$-methyltransferase ( COMT ) genetic polymorphisms and breast cancer susceptibility in a Turkish population. Arch Toxicol. 2002;76:643-9. https://doi.org/10.1007/s00204-002-0387-x.

22. Zanger UM, Schwab M. Cytochrome P450 enzymes in drug metabolism: regulation of gene expression, enzyme activities, and impact of genetic variation. Pharmacol Ther. 2013;138:103-41. https://doi.org/10.1016/J. PHARMTHERA.2012.12.007.

23. Kadlubar FF, Berkowitz GS, Delongchamp RR, et al. The CYP3A4*1B variant is related to the onset of puberty, a known risk factor for the development of breast cancer. Cancer Epidemiol Biomark Prev. 2003; 12:327-31.

24. Miyoshi Y, Ando A, Takamura Y, et al. Prediction of response to docetaxel by CYP3A4 mRNA expression in breast cancer tissues. Int J Cancer. 2002;97: 129-32. https://doi.org/10.1002/ijc.1568.

25. Tsai S-M, Lin C-Y, Wu S-H, et al. Side effects after docetaxel treatment in Taiwanese breast cancer patients with CYP3A4, CYP3A5, and ABCB1 gene polymorphisms. Clin Chim Acta. 2009;404:160-5. https://doi.org/10.1016/J. CCA.2009.03.038.

26. Zhou S-F, Wang B, Yang L-P, Liu J-P. Structure, function, regulation and polymorphism and the clinical significance of human cytochrome P450 1A2. Drug Metab Rev. 2010;42:268-354. https://doi.org/10.3109/0360253 0903286476.

27. Kotsopoulos J, Ghadirian P, El-Sohemy A, et al. The CYP1A2 genotype modifies the association between coffee consumption and breast Cancer risk among BRCA1 mutation carriers. Cancer Epidemiol Biomark Prev. 2007; 16:912-6. https://doi.org/10.1158/1055-9965.EPI-06-1074.

28. Bageman E, Ingvar C, Rose C, Jernstrom H. Coffee consumption and CYP1A2*1F genotype modify age at breast Cancer diagnosis and estrogen receptor status. Cancer Epidemiol Biomark Prev. 2008;17:895-901. https:// doi.org/10.1158/1055-9965.EPI-07-0555.

29. Li J, Ji L. Adjusting multiple testing in multilocus analyses using the eigenvalues of a correlation matrix. Heredity. 2005;95:221-7.

30. Nyholt DR. A simple correction for multiple testing for single-nucleotide polymorphisms in linkage disequilibrium with each other. Am J Hum Genet. 2004;74:765-9.

31. AL-Eitan LN, Jamous RI, Khasawneh RH. Candidate gene analysis of breast Cancer in the Jordanian population of Arab descent: a case-control study. Cancer Investig. 2017;35:256-70. https://doi.org/10.1 080/07357907.2017.1289217.

32. Sadek RF, Zhang LF, Abdul Sater HT. Breast cancer molecular subtypes association with clinical outcomes and race. J Clin Oncol. 2017:35:e12575. https://doi.org/10.1200/JCO.2017.35.15_suppl.e12575.

33. Wang L, Ellsworth KA, Moon I, et al. Functional genetic polymorphisms in the aromatase gene CYP19 vary the response of breast Cancer patients to neoadjuvant therapy with aromatase inhibitors. Cancer Res. 2010;70:319-28. https://doi.org/10.1158/0008-5472.CAN-09-3224.

34. Straume AH, Knappskog S, Lønning PE. Effect of CYP19 rs6493497 and rs7176005 haplotype status on in vivo aromatase transcription, plasma and tissue estrogen levels in postmenopausal women. J Steroid Biochem Mol Biol. 2012;128:69-75. https://doi.org/10.1016/J.JSBMB.2011.08.015.

35. Pineda B, García-Pérez MÁ, Cano A, et al. Associations between aromatase CYP19 rs 10046 polymorphism and breast Cancer risk: from a case-control to a meta-analysis of 20,098 subjects. PLoS One. 2013;8:e53902. https://doi. org/10.1371/journal.pone.0053902.

36. Artigalás O, Vanni T, Hutz MH, et al. Influence of CYP19A1 polymorphisms on the treatment of breast cancer with aromatase inhibitors: a systematic review and meta-analysis. BMC Med. 2015;13:139. https://doi.org/10.1186/ s12916-015-0373-9.

37. Ma X, Qi X, Chen C, et al. Association between CYP19 polymorphisms and breast cancer risk: results from 10,592 cases and 11,720 controls. Breast Cancer Res Treat. 2010;122:495-501. https://doi.org/10.1007/s10549-009-0693-6.

38. Sun M-Y, Du H-Y, Zhu A-N, et al. Genetic polymorphisms in estrogen-related genes and the risk of breast Cancer among Han Chinese women. Int J Mol Sci. 2015;16:4121-35. https://doi.org/10.3390/ijms16024121.

39. Tulsyan S, Agarwal G, Lal P, Mittal B. Significant role of CYP450 genetic variants in cyclophosphamide based breast cancer treatment outcomes: a multi-analytical strategy. Clin Chim Acta. 2014;434:21-8. https://doi.org/10.1 016/J.CCA.2014.04.009

40. MARIE-GENICA. Consortium on Genetic Susceptibility for Menopausal Hormone Therapy Related Breast Cancer Risk. Genetic polymorphisms in phase I and phase II enzymes and breast cancer risk associated with menopausal hormone therapy in postmenopausal women. Breast Cancer Res Treat. 2010;119:463-74. https://doi.org/10.1007/s10549-009-0407-0.

41. Ruiter R, Bijl MJ, van Schaik RH, et al. CYP2C19*2 polymorphism is associated with increased survival in breast cancer patients using tamoxifen. Pharmacogenomics. 2010;11:1367-75. https://doi.org/10.2217/pgs.10.112. 
42. Chamnanphon M, Pechatanan K, Sirachainan E, et al. Association of CYP2D6 and CYP2C19 polymorphisms and disease-free survival of Thai post-menopausal breast cancer patients who received adjuvant tamoxifen. Pharmgenomics Pers Med. 2013;6:37-48. https://doi.org/1 $0.2147 /$ PGPM.S42330.

43. van Schaik RH, Kok M, Sweep FC, et al. The CYP2C19*2 genotype predicts tamoxifen treatment outcome in advanced breast cancer patients. Pharmacogenomics. 2011;12:1137-46. https://doi.org/10.2217/pgs.11.54.

44. Long J-R, Cai Q, Shu X-O, et al. Genetic polymorphisms in estrogenmetabolizing genes and breast cancer survival. Pharmacogenet Genomics. 2007;17:331-8. https://doi.org/10.1097/FPC.0b013e32801a3bfe.

45. Sangrajrang S, Sato Y, Sakamoto H, et al. Genetic polymorphisms of estrogen metabolizing enzyme and breast cancer risk in Thai women. Int J Cancer. 2009;125:837-43. https://doi.org/10.1002/ijc.24434.

46. Wang H, Zhang Z, Han S, et al. CYP1A2 rs762551 polymorphism contributes to cancer susceptibility: a meta-analysis from 19 case-control studies. BMC Cancer. 2012;12:528. https://doi.org/10.1186/1471-2407-12-528.

47. Tian Z, Li Y-L, Zhao L, Zhang C-L. Role of CYP1A2*1F polymorphism in cancer risk: evidence from a meta-analysis of 46 case-control studies. Gene. 2013;524:168-74. https://doi.org/10.1016/J.GENE.2013.04.038.

48. Qiu L-X, Yao L, Mao C, et al. Lack of association of CYP1A2-164 a/C polymorphism with breast cancer susceptibility: a meta-analysis involving 17,600 subjects. Breast Cancer Res Treat. 2010;122:521-5. https://doi.org/10.1 007/s10549-009-0731-4.

49. Lam SW, Frederiks CN, van der Straaten T, et al. Genotypes of CYP2C8 and FGD4 and their association with peripheral neuropathy or early dose reduction in paclitaxel-treated breast cancer patients. Br J Cancer. 2016;115: 1335-42. https://doi.org/10.1038/bjc.2016.326.

50. Hertz DL, Kidwell KM, Seewald NJ, et al. Polymorphisms in drugmetabolizing enzymes and steady-state exemestane concentration in postmenopausal patients with breast cancer. Pharmacogenomics J. 2017;17: 521-7. https://doi.org/10.1038/tpj.2016.60.

51. Pascual T, Apellániz-Ruiz M, Pernaut C, et al. Polymorphisms associated with everolimus pharmacokinetics, toxicity and survival in metastatic breast cancer. PLoS One. 2017:12:e0180192. https:/doi.org/10.1371/journal.pone.0180192.

52. Huang Y, Trentham-Dietz A, García-Closas M, et al. Association of CYP1B1 haplotypes and breast cancer risk in Caucasian women. Cancer Epidemiol Biomark Prev. 2009;18:1321-3. https://doi.org/10.1158/1055-9965.EPI-08-0853.

53. Gaudet MM, Chanock S, Lissowska J, et al. Genetic variation of cytochrome P450 1B1 (CYP1B1) and risk of breast cancer among polish women. Pharmacogenet Genomics. 2006;16:547-53. https://doi.org/10.1097/01.fpc. $0000215067.29342 .6 f$

\section{Publisher's Note}

Springer Nature remains neutral with regard to jurisdictional claims in published maps and institutional affiliations.

Ready to submit your research? Choose BMC and benefit from:

- fast, convenient online submission

- thorough peer review by experienced researchers in your field

- rapid publication on acceptance

- support for research data, including large and complex data types

- gold Open Access which fosters wider collaboration and increased citations

- maximum visibility for your research: over $100 \mathrm{M}$ website views per year

At $\mathrm{BMC}$, research is always in progress.

Learn more biomedcentral.com/submissions 